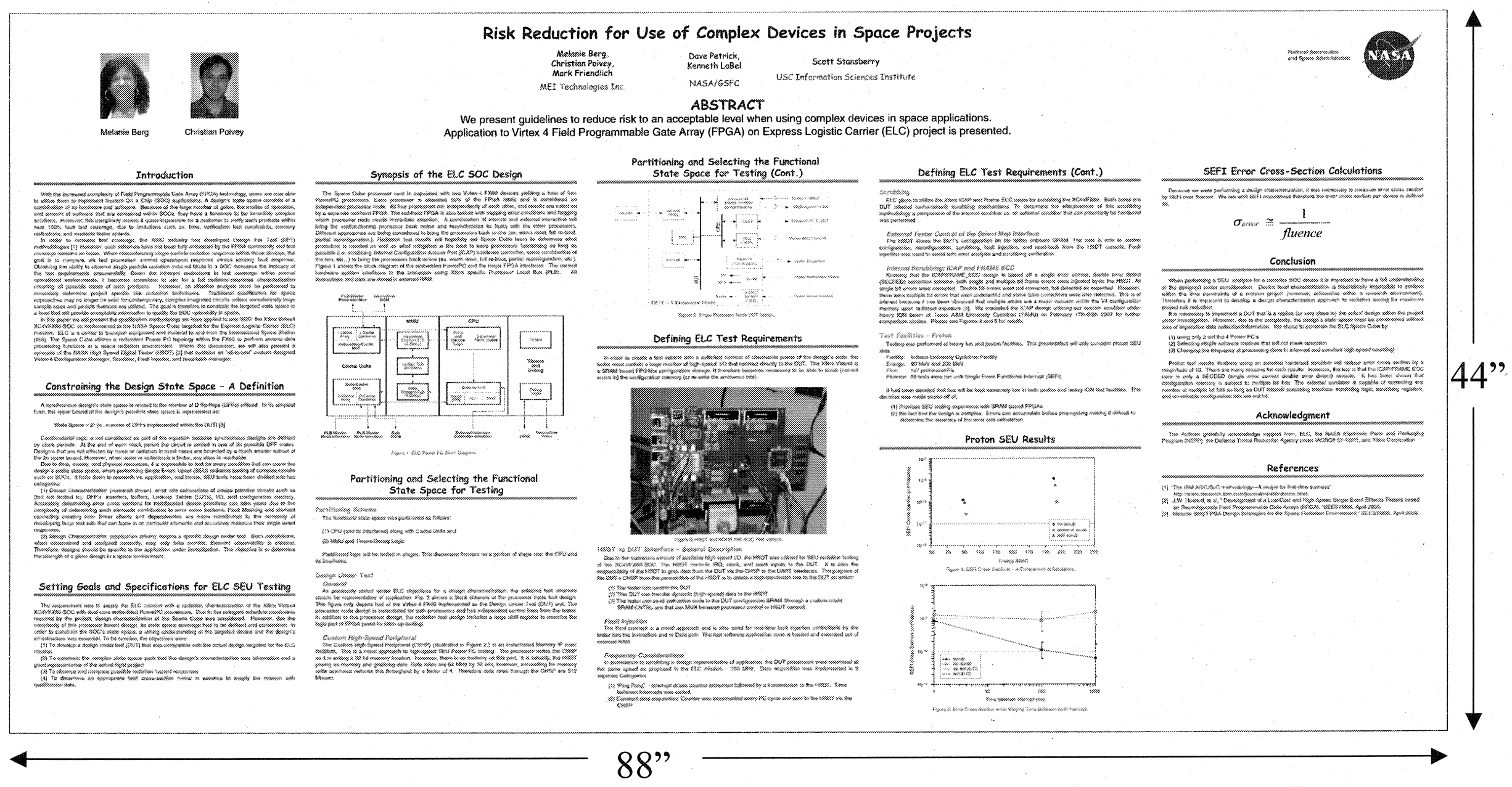

To be presented by Melanie Berg at the IEEE Nuclear and Space Radiation Effects Conference (NSREC), July 23-27, 2007 and to be published in the 2007 IEEE Radiation Effects Poster Session and on http://rahome.gsfc.nasa.gov 


\title{
Risk Reduction for Use of Complex Devices in Space Projects
}

\author{
Melanie Berg, \\ Christian Poivey, \\ Mark Friendlich \\ MEI Technologies Inc.
}

Dave Petrick,

Kenneth LaBel

NASA/GSFC

Scott Stansberry

USC Information Sciences Institute

ABSTRACT

We present guidelines to reduce risk to an acceptable level when using complex devices in space applications.

Application to Virtex 4 Field Programmable Gate Array (FPGA) on Express Logistic Carrier (ELC) project is presented. 


\section{Introduction}

With the increased complexity of Field Programmable Gate Array (FPGA) technology, users are now able to utilize them to implement System On a Chip (SOC) applications. A design's state space consists of a combination of its hardware and software. Because of the large number of gates, the modes of operation, and amount of software that are contained within SOCs, they have a tendency to be incredibly complex solutions. However, this complexity makes it quasi-impossible for a customer to verify such products within near $100 \%$ fault test coverage, due to limitations such as: time, verification tool constraints, memory restrictions, and available tester speeds.

In order to increase test coverage, the ASIC industry has developed Design For Test (DFT) methodologies [1]. However, such schemes have not been fully embraced by the FPGA community and test coverage remains an issue. When characterizing single particle radiation response within these devices, the goal is to compare, via test processes, normal operational response versus ionizing fault response. Obtaining the ability to observe single particle radiation-induced faults in a SOC increases the intricacy of the test requirements exponentially. Given the inherent restrictions in test coverage within normal operational environments, it becomes unrealistic to aim for a full radiation-response characterization covering all possible states of such products. However, an effective analysis must be performed to accurately determine project specific risk reduction techniques. Traditional qualification for space approaches may no longer be valid for contemporary, complex integrated circuits unless unrealistically large sample sizes and particle fluences are utilized. The goal is therefore to constrain the targeted state space to a level that will provide acceptable information to qualify the SOC operability in space.

In this paper we will present the qualification methodology we have applied to one SOC: the Xilinx Virtex4 XC4VFX60-SOC as implemented in the NASA Space Cube targeted for the Express Logistic Carrier (ELC) mission. ELC is a carrier to transport equipment and material to and from the International Space Station (ISS). The Space Cube utilizes a redundant Power PC topology within the FX60 to perform several data processing functions in a space radiation environment. Within this discussion, we will also present a synopsis of the NASA High Speed Digital Tester (HSDT) [2] that contains an "all-in-one" custom designed Virtex-4 Configuration Manager, Scrubber, Fault Injector, and read-back manager.

To be presented by Melanie Berg at the IEEE Nuclear and Space Radiation Effects Conference (NSREC), July 23-27, 


\section{Constraining the Design State Space - A Definition}

A synchronous design's state space is related to the number of D flip-flops (DFFs) utilized. In its simplest form, the upper bound of the design's possible state space is represented as:

$$
\text { State Space }=2^{n}(n: \text { number of DFFs implemented within the DUT) [3] }
$$

Combinatorial logic is not considered as part of the equation because synchronous designs are defined by clock periods. At the end of each clock period the circuit is settled in one of $2 n$ possible DFF states. Design's that are not effected by noise or radiation in most cases are bounded by a much smaller subset of the $2 n$ upper bound. However, when noise or radiation is a factor, any state is reachable.

Due to time, money, and physical resources, it is impossible to test for every condition that can cover the design's entire state space, when performing Single Event Upset (SEU) radiation testing of complex circuits such as SOCs. It boils down to research vs. application, and hence, SEU tests have been divided into two categories:

(1) Device Characterization (research driven): error rate calculations of device primitive circuits such as (but not limited to): DFF's, inverters, buffers, Look-up Tables (LUTs), I/O, and configuration memory. Accurately determining error cross sections for multifaceted device primitives can take years due to the complexity of determining each elements contribution to error cross sections. Fault Masking and element cascading creating non- linear effects and dependencies are major contributors to the necessity of developing large test sets that can hone in on particular elements and accurately measure their single event responses.

(2) Design Characterization (application driven): targets a specific design under test. Such calculations, when constrained and analyzed correctly, may only take months. Element observability is minimal. Therefore, designs should be specific to the application under investigation. The objective is to determine the strength of a given design in a space environment. 


\section{Setting Goals and Specifications for ELC SEU Testing}

The requirement was to supply the ELC mission with a radiation characterization of the Xilinx Virtex4 XC4VFX60-SOC with dual-core embedded PowerPC processors. Due to the stringent schedule constraints required by the project, design characterization of the Space Cube was considered. However, due the complexity of this processor based design, its state space coverage had to be defined and constrained. In order to constrain the SOC's state space, a strong understanding of the targeted device and the design's infrastructure was essential. To be concise, the objectives were:

(1) To develop a design under test (DUT) that was compatible with the actual design targeted for the ELC mission

(2) To constrain the complex state space such that the design's characterization was informative and a good representative of the actual flight project

(3) To observe and compare possible radiation hazard responses

(4) To determine an appropriate fault cross-section metric in essence to supply the mission with qualification data.

To be presented by Melanie Berg at the IEEE Nuclear and Space Radiation Effects Conference (NSREC), July 23-27, 2007 and to be published in the 2007 IEEE Radiation Effects Poster Session and on http://rahome.gsfc.nasa.gov 


\section{Synopsis of the ELC SOC Design}

The Space Cube processor card is populated with two Virtex-4 FX60 devices yielding a total of four PowerPC processors. Each processor is allocated $50 \%$ of the FPGA fabric and is considered an independent processor node. All four processors run independently of each other, and results are voted on by a separate rad-hard FPGA. The rad-hard FPGA is also tasked with trapping error conditions and flagging which processor node needs immediate attention. A combination of internal and external interaction will bring the malfunctioning processor back online and resynchronize its tasks with the other processors. Different approaches are being considered to bring the processors back online (ex. warm reset, full re-boot, partial reconfiguration.). Radiation test results will hopefully aid Space Cube team to determine what procedure is needed as well as what mitigation is the best to keep processors functioning as long as possible (i.e. scrubbing, Internal Configuration Access Port (ICAP) hardware controller, some combination of the two, etc...) to bring the processors back online (ex. warm reset, full re-boot, partial reconfiguration, etc.). Figure 1 shows the block diagram of the embedded PowerPC and the major FPGA interfaces. The current hardware system interfaces to the processor using Xilinx specific Processor Local Bus (PLB). All instructions and data are stored in external RAM. 


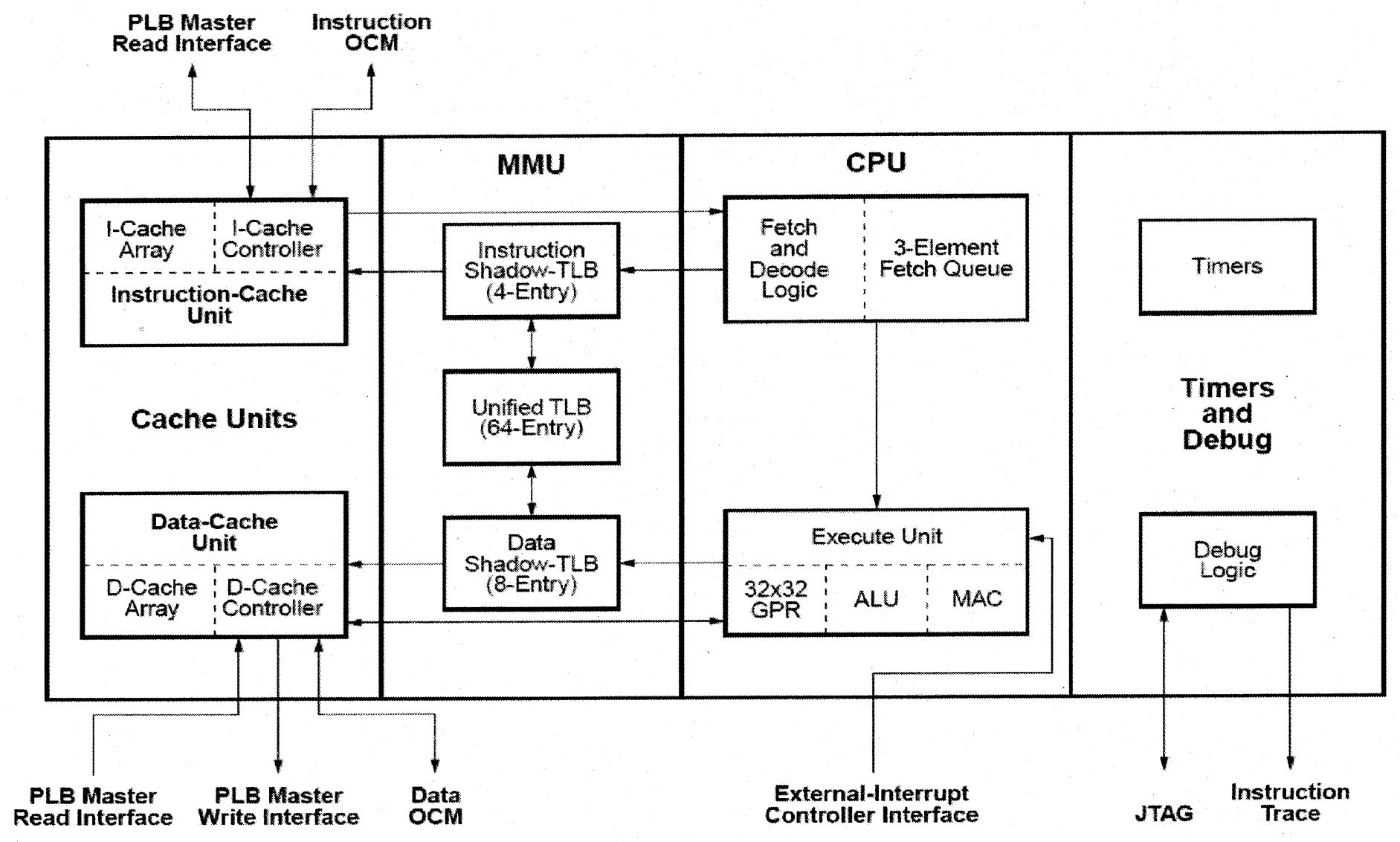

Figure 1: ELC Power PC Block Diagram.

To be presented by Melanie Berg at the IEEE Nuclear and Space Radiation Effects Conference (NSREC), July 23-27, 


\section{Partitioning and Selecting the Functional State Space for Testing}

\section{Partitioning Scheme}

The functional state space was partitioned as follows:

(1) CPU (and its interfaces) along with Cache Units and

(2) MMU and Timers/Debug Logic.

Partitioned logic will be tested in stages. This discussion focuses on a portion of stage one: the CPU and its interfaces.

\section{Design Under Test}

\section{General}

As previously stated under ELC objectives for a design characterization, the selected test structure should be representative of application. Fig. 2 shows a block diagram of the processor node test design. This figure only depicts half of the Virtex-4 FX60 implemented as the Design Under Test (DUT) unit. The processor node design is instantiated for both processors and has independent control lines from the tester. In addition to this processor design, the radiation test design includes a large shift register to exercise the logic part of FPGA (used for latch-up testing).

\section{Custom High-Speed Peripheral}

The Custom High-Speed Peripheral (CSHP) (illustrated in Figure 2.) is an instantiated Memory IP core: $8 \times 32$ bits. This is a novel approach to high-speed SEU Power PC testing. The processor writes the CSHP as it is writing a 32 bit memory location. However, there is no memory on this port. It is actually, the HSDT posing as memory and grabbing data. Data rates are $64 \mathrm{MHz}$ by 32 bits, however, accounting for memory write overhead reduces this throughput by a factor of 4 . Therefore data rates through the CHSP are 512 $\mathrm{Mb} / \mathrm{sec}$. 


\section{Partitioning and Selecting the Functional State Space for Testing (Cont.)}

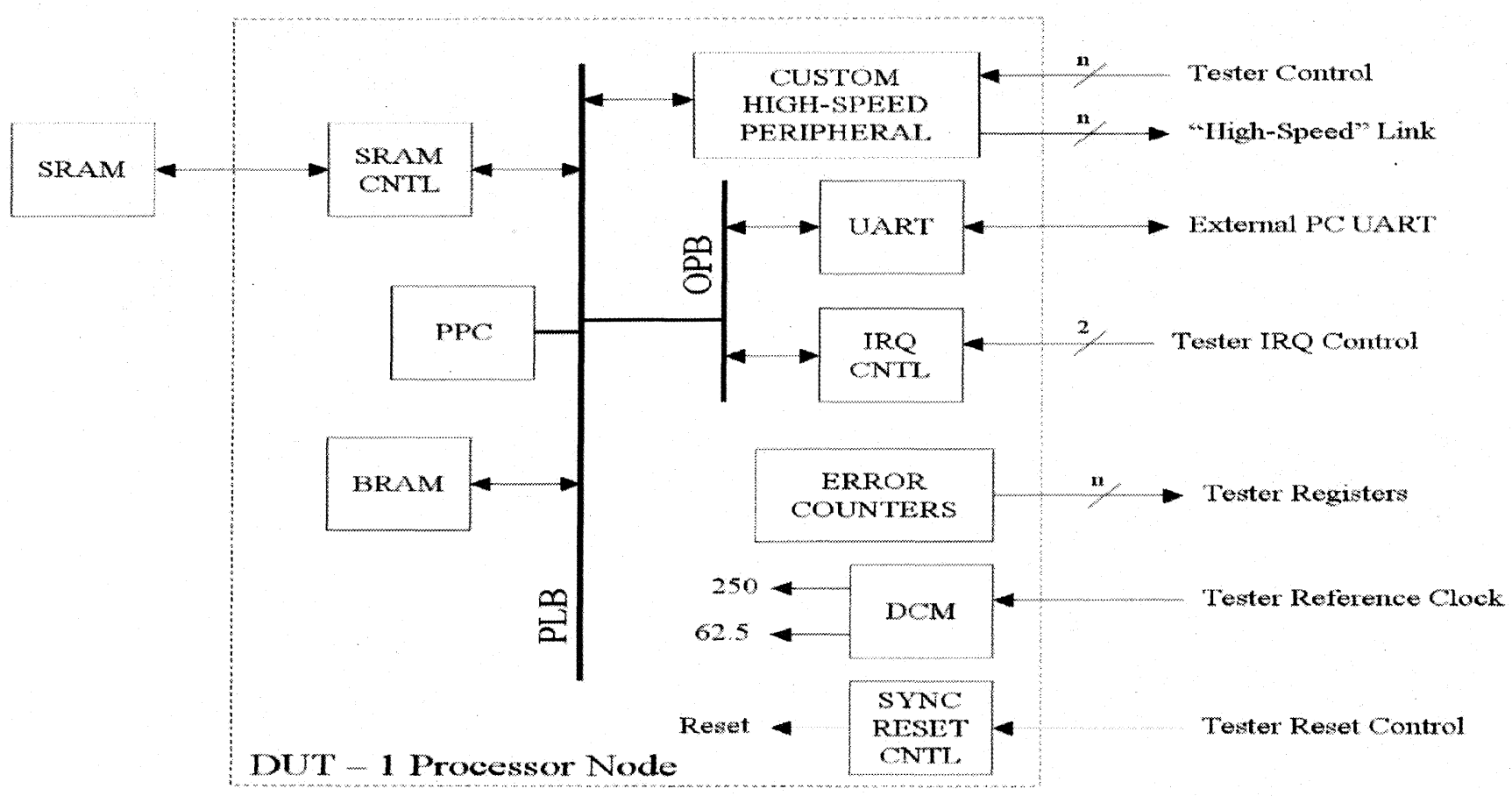

Figure 2: Single Processor Node DUT design. 


\section{Defining ELC Test Requirements}

In order to create a test vehicle with a sufficient number of observable points of the design's state, the tester must contain a large number of high-speed I/O that connect directly to the DUT. The Xilinx Virtex4 is a SRAM based FPGAfor configuration storage. It therefore becomes necessary to be able to scrub (correct errors in) the configuration memory (or re-write the erroneous bits).

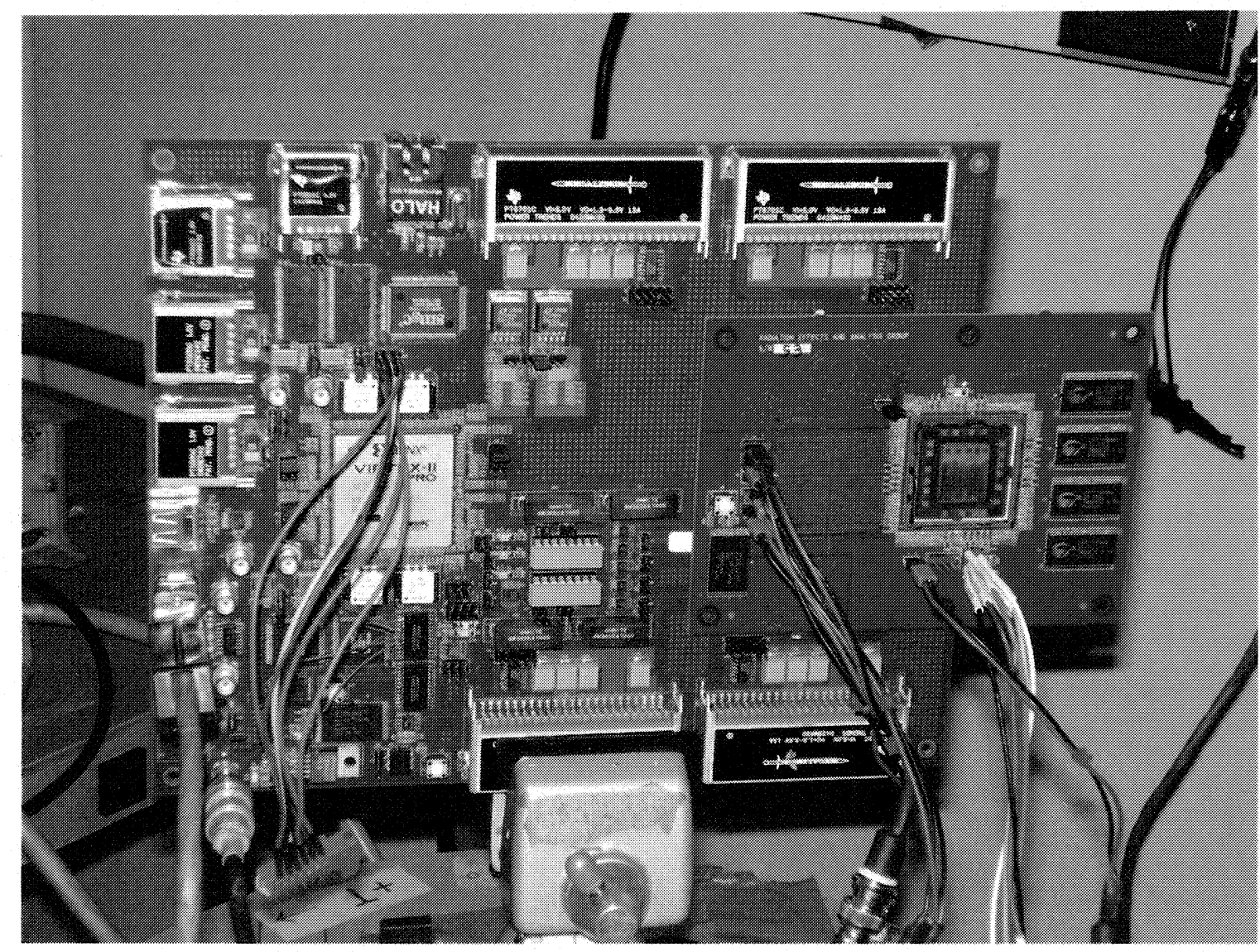

Figure 3: HSDT and XC4VFX60-SOC Test Vehicle.

To be presented by Melanie Berg at the IEEE Nuclear and Space Radiation Effects Conference (NSREC), July 23-27, 2007 and to be published in the 2007 IEEE Radiation Effects Poster Session and on http://rahome.gsfc.nasa.gov 


\section{HSDT to DUT Interface - General Description}

Due to the numerous amount of available high-speed I/O, the HSDT was utilized for SEU radiation testing of the XC4VFX60-SOC. The HSDT controls IRQ, clock, and reset inputs to the DUT. It is also the responsibility of the HSDT to grab data from the DUT via the CHSP or the UART interfaces. The purpose of the DUT's CHSP from the perspective of the HSDT is to create a high-bandwidth bus to the DUT on which:

(1) The tester can control the DUT

(2) Tthe DUT can transfer dynamic (high-speed) data to the HSDT

(3) The tester can send instruction code to the DUT configuration SRAM (through a custom made SRAM CNTRL unit that can MUX between processor control or HSDT control).

\section{Fault Injection}

The third concept is a novel approach and is also used for real-time fault injection controllable by the tester into the instruction and or Data path. The test software application code is loaded and executed out of external RAM.

\section{Frequency Considerations}

In accordance to emulating a design representative of application, the DUT processors were exercised at the same speed as proposed in the ELC mission - $250 \mathrm{MHz}$. Data acquisition was implemented in 2 separate Categories:

(1) "Ping Pong" - Interrupt driven counter increment followed by a transmission to the HSDT. Time between Interrupts was varied.

(2) Constant data acquisition: Counter was incremented every PC cycle and sent to the HSDT via the CHSP 


\section{Defining ELC Test Requirements (Cont.)}

\section{Scrubbing}

ELC plans to utilize the Xilinx ICAP and Frame ECC cores for scrubbing the XC4VFX60. Such cores are DUT internal (unhardened) scrubbing mechanisms. To determine the effectiveness of this scrubbing methodology a comparison of the internal scrubber vs. an external scrubber that can potentially be hardened was performed

\section{External Tester Control of the Select Map Interface}

The HSDT stores the DUT's configuration bit file within onboard SRAM. The user is able to control configuration, reconfiguration, scrubbing, fault injection, and read-back from the HSDT console. Fault injection was used to assist with error analysis and scrubbing verification.

\section{Internal Scrubbing: ICAP and FRAME ECC}

Knowing that the ICAP/FRAME_ECC design is based off a single error correct, double error detect (SECDED) correction scheme, both single and multiple bit frame errors were injected byvia the HSDT. All single bit errors were corrected. Double bit errors were not corrected, but detected as expected. However, there were multiple bit errors that went undetected and some false corrections were also detected. This is of interest because it has been observed that multiple errors are a major concern within the $\mathrm{V} 4$ configuration memory upon radiation exposure [3]. We irradiated the ICAP design utilizing our custom scrubber under heavy ION beam at Texas A\&M University Cyclotron (TAMU) on February 17th-20th 2007 for further comparison studies. Please see Figures 4 and 5 for results. 


\section{Test Facilities - Proton}

Testing was performed at heavy lon and proton facilities. This presentation will only consider proton SEU data.

Facility: Indiana University Cyclotron Facility.

Energy: $93 \mathrm{MeV}$ and $200 \mathrm{MeV}$

Flux: $\quad 1 \mathrm{e} 7 \mathrm{protons} / \mathrm{cm}^{2} / \mathrm{s}$

Fluence: All tests were run until Single Event Functional Interrupt (SEFI).

It had been decided that flux will be kept extremely low in both proton and heavy ION test facilities. This decision was made based off of:

(1) Previous SEU testing experience with SRAM based FPGAs

(2) the fact that the design is complex. Errors can accumulate before propagating making it difficult to determine the accuracy of the error rate calculation. 


\section{Proton SEU Results}

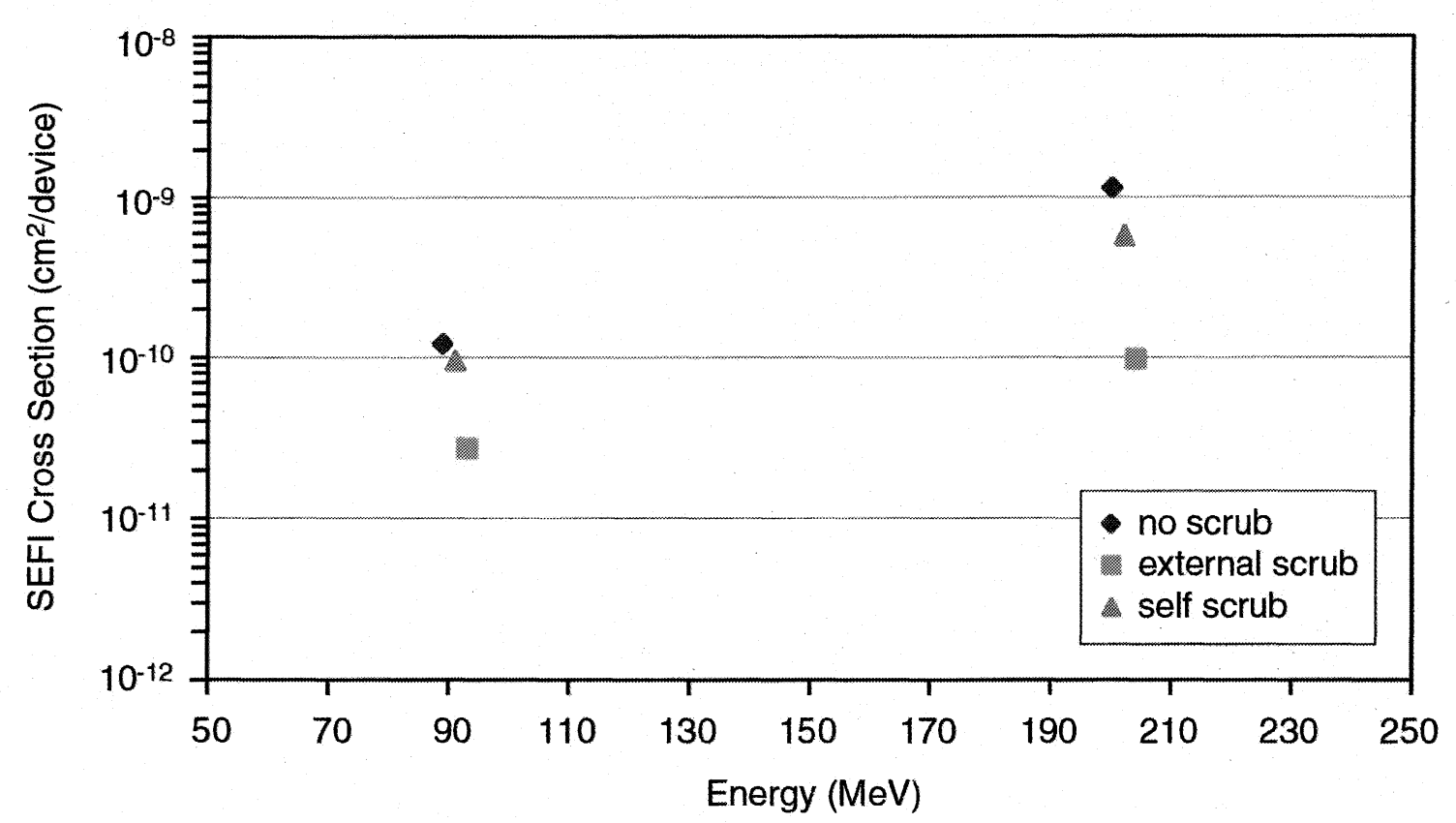

Figure 4: SEFI Cross Sections - A Comparison of Scrubbers. 


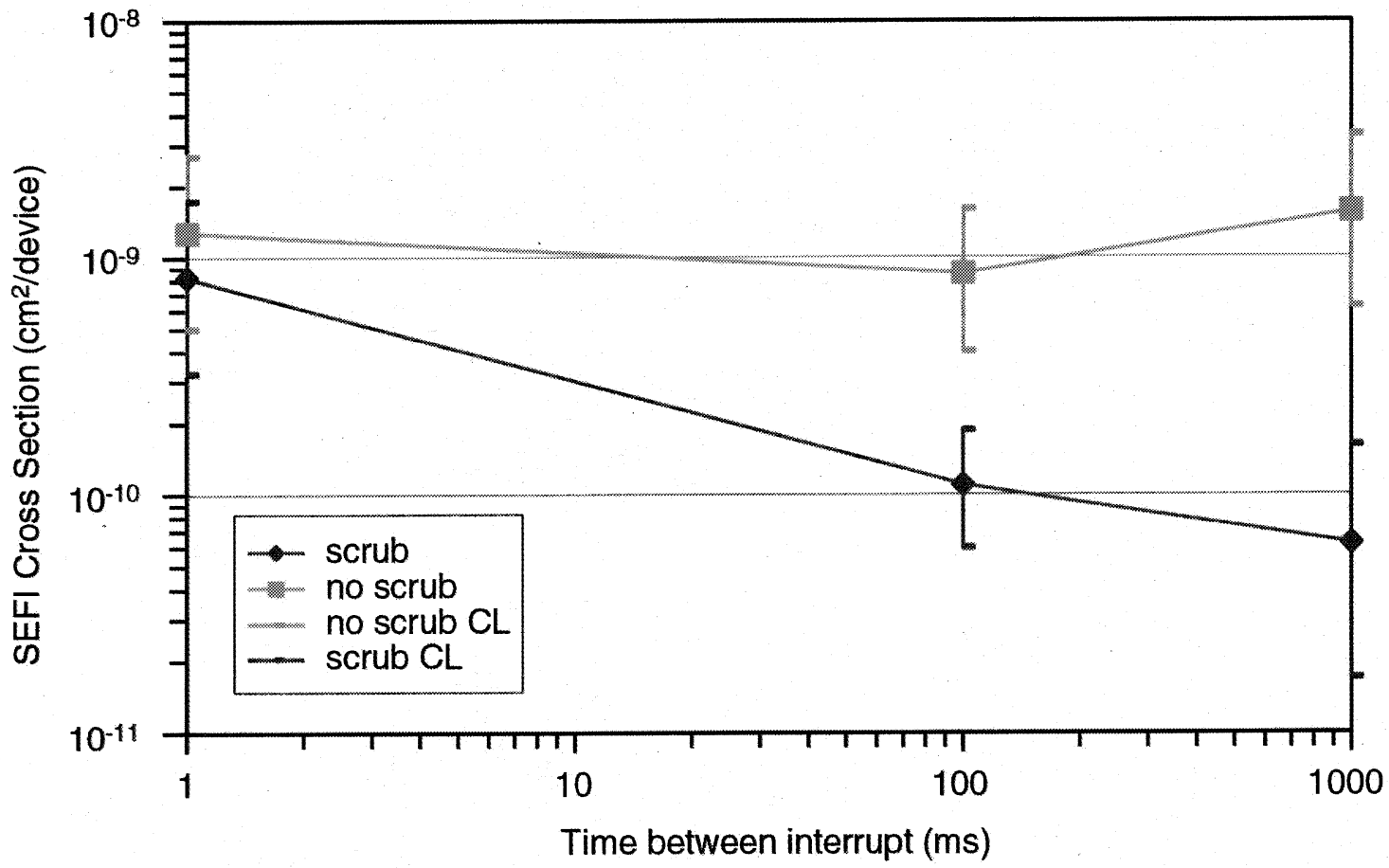

Figure 5: Error Cross-Section while Varying Time Between each Interrupt. 


\section{SEFI Error Cross-Section Calculations}

Because we were performing a design characterization, it was necessary to measure error cross section by SEFI over fluence. We ran until SEFI occuranvce therefore the error cross section per device is defined as:

$$
\sigma_{\text {error }} \cong \frac{1}{\text { fluence }}
$$




\section{Conclusion}

When performing a SEU analysis for a complex SOC device it is important to have a full understanding of the design(s) under consideration. Device level characterization is theoretically impossible to achieve within the time constraints of a mission project (however, achievable within a research environment). Therefore it is important to develop a design characterization approach to radiation testing for maximum project risk reduction.

It is necessary to implement a DUT that is a replica (or very close to) the actual design within the project under investigation. However, due to the complexity, the design's state space must be constrained without loss of imperative data collection/information. We chose to constrain the ELC Space Cube by:

(1) using only 2 out the 4 Power PC's

(2) Selecting simple software routines that will not mask operation

(3) Changing the frequency of processing (time to interrupt and constant high speed counting)

Proton test results illustrate using an external hardened scrubber will reduce error cross section by a magnitude of 10. There are many reasons for such results. However, the key is that the ICAP/FRAME ECC core is only a SECDED (single error correct double error detect) module. It has been shown that configuration memory is subject to multiple bit hits. The external scrubber is capable of correcting any number of multiple bit hits as long as DUT internal scrubbing interface, scrubbing logic, scrubbing registers, and un-writable configuration bits are not hit. 


\section{Acknowledgment}

The Authors gratefully acknowledge support from, ELC, the NASA Electronic Parts and Packaging Program (NEPP), the Defense Threat Reduction Agency under IACRO\# 07-4207l, and Xilinx Corporation.

\section{References}

[1] "The IBM ASIC/SoC methodology-A recipe for first-time success" http://www.research.ibm.com/journal/rd/466/doerre.html.

[2] J.W. Howard, et al, " Development of a Low-Cost and High-Speed Single Event Efftects Testers based on Reconfigurable Field Programmable Gate Arrays (FPGA), "SEESYM06, April 2006.

[3] Melanie Berg"FPGA Design Strategies for the Space Radiation Environment," SEESYM06, April 2006. 